\title{
Data Processing in Software Defined Radio (SDR) based Multiband Transceiver
}

\author{
Amit Naik \\ Communication Laboratory, Electronics Engineering \\ Defence Institute of Advanced Technology, \\ DRDO Lab \\ Girinagar, Pune 411025
}

\begin{abstract}
Different types of wireless communication technologies use different wireless frequency bands. We are experiencing many working tasks at same time. In this paper data processing for various frequency bands in a multiband transceiver is elucidate. Different types of input data are created by vector signal generator (SMU200A) with the frequency range of $100 \mathrm{KHz}$ to 3GHz.Data is effectively communicated through multiband transceiver Simulink model. At the receiver end data can be successfully recovered by linking through the Real-time signal analyzer (RSA6114B)(9KHz- 14GHz) or using RTL 820T2 SDR Dongle with the frequency range of $25 \mathrm{MHz}$ to $1800 \mathrm{MHz}$ with a span of $2.5 \mathrm{MHz}$.Proposed multiband transceiver is proficient of transmitting and receiving data at different frequencies. Lastly, the outcomes acquired are presented in the graphical system.
\end{abstract}

\section{Keywords}

Data Processing; Frequency; Multiband; Software Defined Radio (SDR); Transceiver

\section{INTRODUCTION}

The region of wireless communication structure has perceived exponential progression in the previous decade. Nowadays every electronic communication system is moving from wired to the wireless system and becoming a smart wireless system [10]. Frequently mobile service provider's launches innovative cutting-edge devices, applications and utilities for live television, audio and video calling, file sharing and transferring, etc.

\author{
K. Krishna Naik, PhD \\ Communication Laboratory, Electronics Engineering \\ Defence Institute of Advanced Technology, \\ DRDO Lab \\ Girinagar, Pune 411025
}

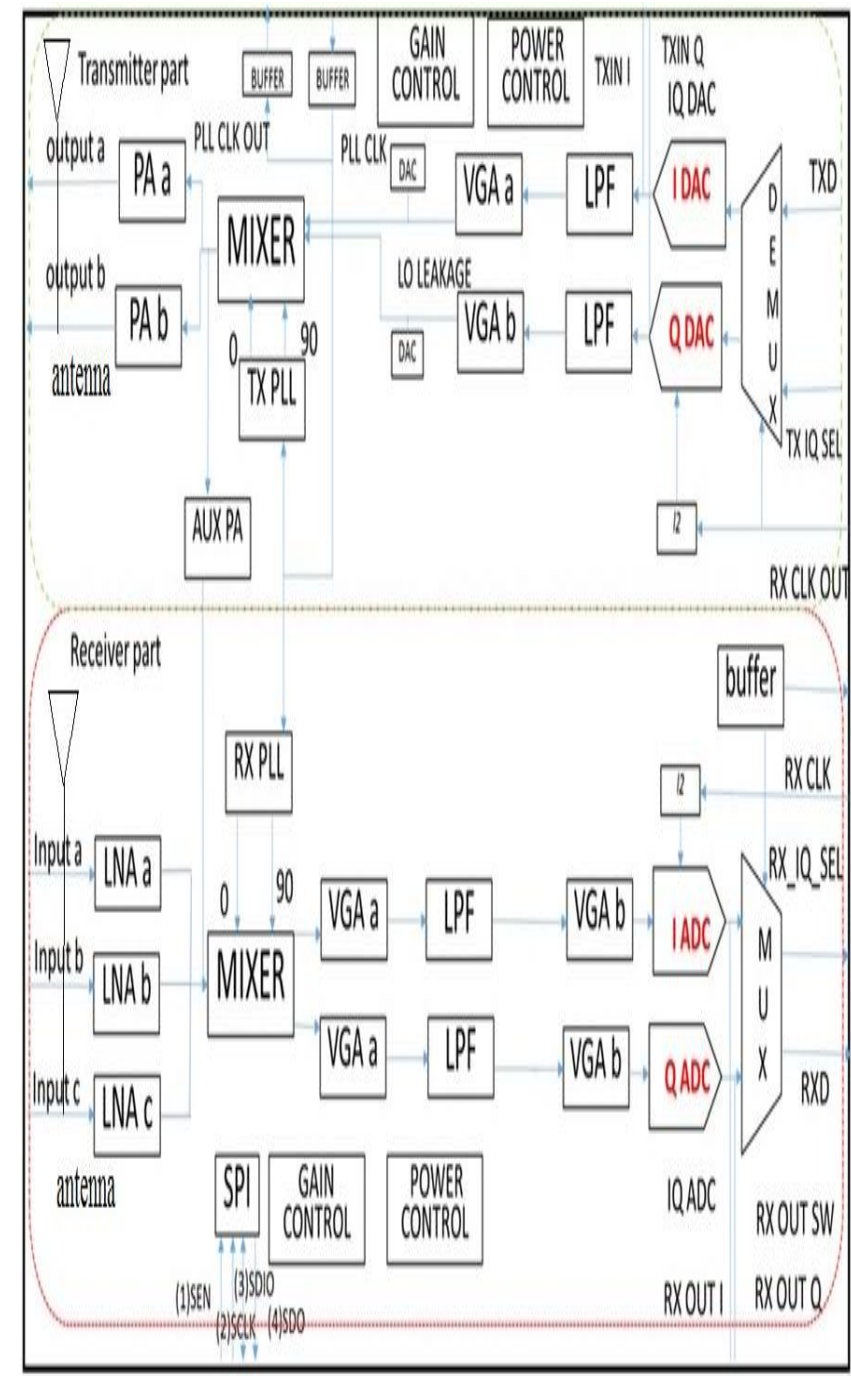

Figure1: Functional Block diagram of Transceiver [5]

An application runs effortlessly if sufficient frequency is assigned to service providers. The number of wireless communication consumers is increasing exponentially, but the resources and bandwidth allocation for each technology is restricted.

It affects the speed of the network, data transfer rate, jamming in the setup, call drop and numerous added problems in wireless communication. Different frequency bands are assigned to different facilities, for example Bluetooth, WLAN, CDMA and GSM are communication technologies which function on different frequency bands [9]. For usage of all the technologies by a single unit, it should be a smart unit which is proficient of 
handling of multiple data processing at different frequencies [8]. Hence here is a design trade-off among the application diversity and the dimensions minimization of consumer device [1].

The combination of transmitter and receiver in a single package, in which hardware is cooperatively used by the transmitter and receiver is jointly recognized as the transceiver [3]. If multiple bands of frequencies are used for wireless communication then we can call it as multiband operation [4].Keeping in view of all these challenges confronted in the design, this is a good model of dynamic data processing in multiband transceiver [2].

\section{COMMUNICATION TECHNOLOGIES}

Wireless communication uses different communication technologies at different frequencies, most standard technologies are

Bluetooth: Bluetooth is a low cost, low power standardized protocol. It is a protected protocol impeccable for short- range wireless data transfer between two electronic systems. It uses 12 digits hexadecimal value with unique 48-bit address, in which first 24 bits is organization unique identifier (OUI) which is used to identify manufacturer and last 24 bits is unique part of address, user can change this address to name or other identification for security and easy identification for known user. It is extensively used in consumer products and applications like wireless head phone, mouse etc. its main benefit is that it is free to use.

Wireless Local Area Network (WLAN): It is a packet data communication network which offers higher bandwidth and it is less expensive for a restricted local geographical region with flexibility, mobility, portability and easy installation capability. Benefit of the WLAN is that no cable link is required. Its main disadvantages are Signal Bleed Over, less capacity and overpriced.

Code Division Multiple Access (CDMA): It uses special codes which are assigned by user to transmitter, each bit ( 0 or 1$)$ is spread into pulses per chip so that the user at receiver end which knows the sending pattern will only be able to recover the unique message and other users will receive noise in the form of ripples. Benefits of using CDMA are that it can effortlessly handle data and voice, larger capacity, greater strength against frequency selective fading and Inter-Symbol Interference (ISI). It uses soft hand off with enhanced power control and offers superior confidentiality. Its shortcomings are complexity, intricate power control and limited bandwidth for each user.

Global System for Mobile Communication: Broadly used second generation (2G) worldwide digital cellular telecommunication system with roaming competence [7]. It uses digital Time division multiple access (TDMA) which offers secrecy, greater capacity and space for more number of users within existing bandwidth. It offers speech, voice, short message service (SMS), data transfer like teletex, facsimile, videotext with bi-directional transfer capability. It has store and forward function (at) very low cost [7].It offers good speech quality. It uses $200 \mathrm{kHz}$ radio frequency (RF) channels [9].
Table 1: Frequency details of Bluetooth, WLAN, CDMA and GSM and proposed unit [6][9]

\begin{tabular}{|c|c|c|c|}
\hline & Transmitter band & Receiver band & Channel spacing \\
\hline ВLUETOOTH & $2.402-2.4181 \mathrm{GHz}$ & $\begin{array}{c}2.402-2.4181 \\
\mathrm{GHz}\end{array}$ & $1 \mathrm{MHz}$ \\
\hline WLAN & $2.400-2.485 \mathrm{GHz}$ & $\begin{array}{c}2.400-2.485 \\
\mathrm{GHz}\end{array}$ & 14(overlapping channels) \\
\hline CDMA & $1920-1980 \mathrm{MHz}$ & $2110-2170 \mathrm{MHz}$ & $5 \mathrm{MHz}$ \\
\hline GSM & $880-915 \mathrm{MHz}$ & $925-960 \mathrm{MHz}$ & $200 \mathrm{KHz}$ \\
\hline Proposed Unit & $880 \mathrm{MHz}-2.485 \mathrm{GHz}$ & $\begin{array}{c}925 \mathrm{MHz}-2.485 \\
\mathrm{GHz}\end{array}$ & $\begin{array}{l}\text { "(depends upon } \\
\text { technology selected) }\end{array}$ \\
\hline
\end{tabular}

\section{DATA PROCESSING IN MULTIBAND TRANSCEIVER}

Data is generated by Vector signal generator (SMU200A) with the frequency range of $100 \mathrm{KHz}$ to $3 \mathrm{GHz}$. We can modify input data, modulation, shape and other parameters. Microstrip patch antenna is used for data transmission. Data is now transmitted through multiband transceiver Simulink model and received using RTL 820T2 SDR Dongle. We can receive data by using RTL820T2 SDR dongle its frequency range is $25 \mathrm{MHz}$ to 1800 $\mathrm{MHz}$ with a span of $2.5 \mathrm{MHz}$.

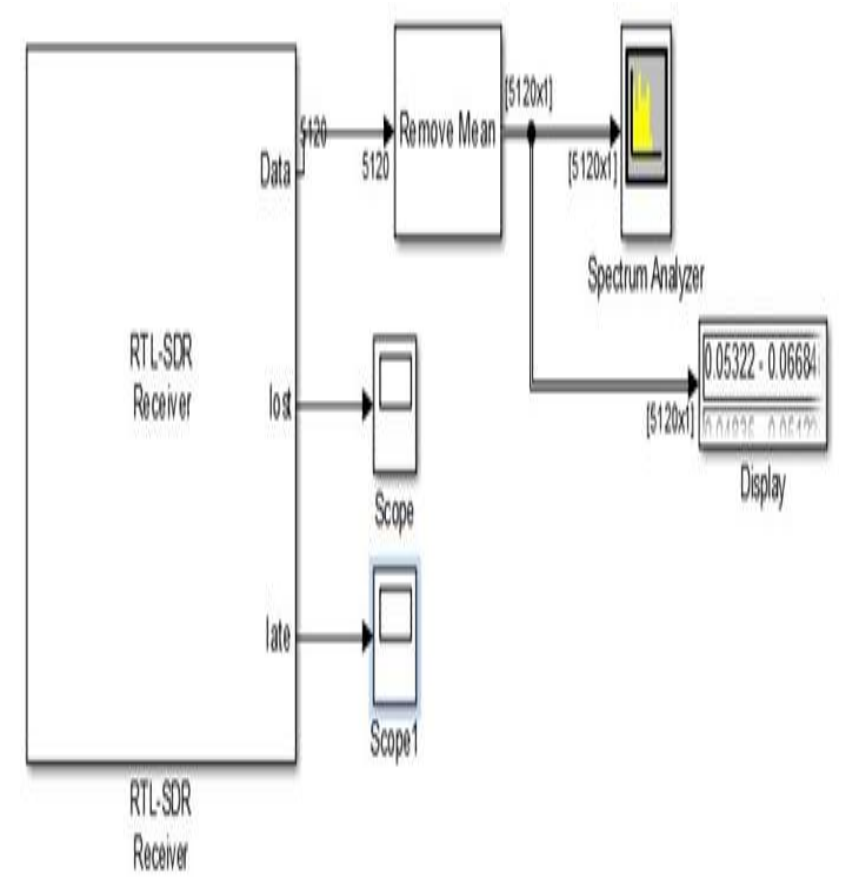




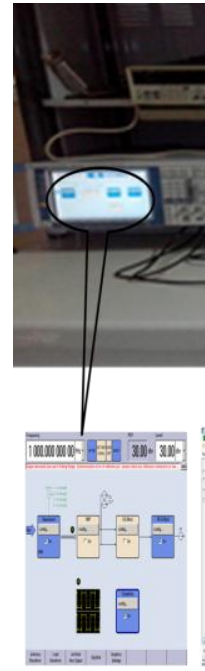

a)

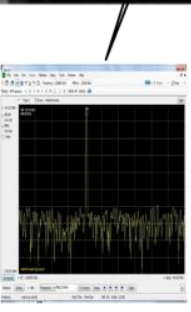

b)
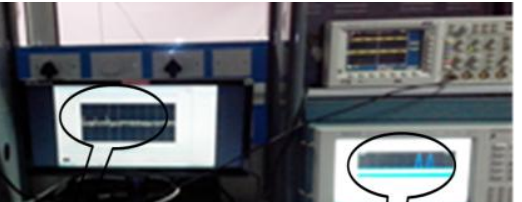
.

政
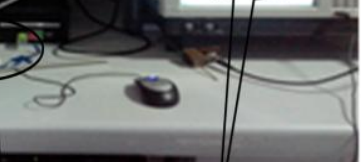

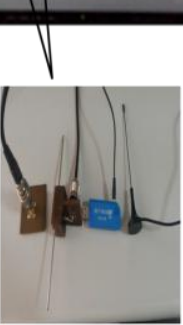

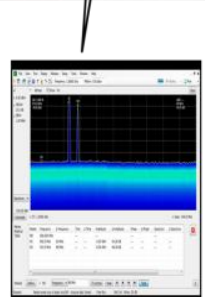

d)
Figure 2: Experimental setup for data processing. Figure 2(a) Shows the vector signal generator, 2(b) explains output waveform of SDR, figure 2(c) shows antennae and SDR module, figure 2(d) shows output of spectrum analyzer

\section{EXPERIMENTAL RESULTS AND DISCUSSION}

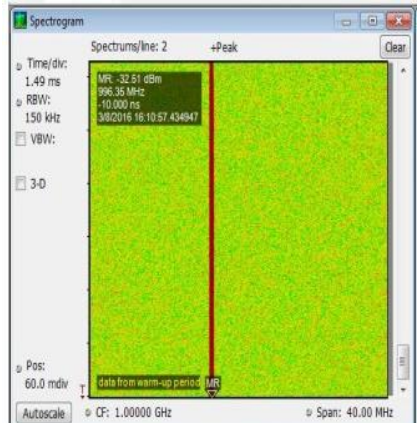

(a)

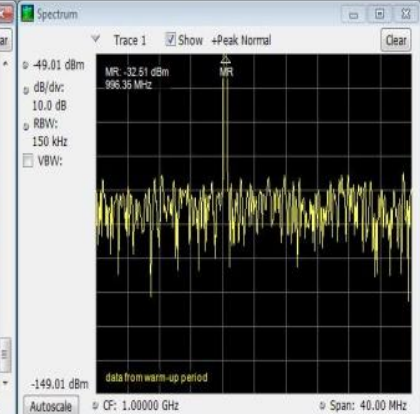

(b)

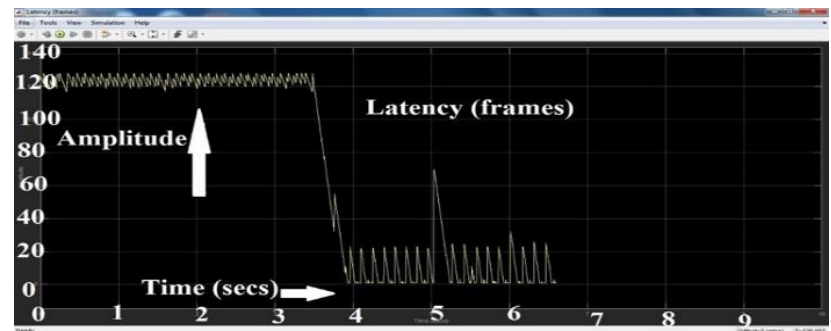

(c)

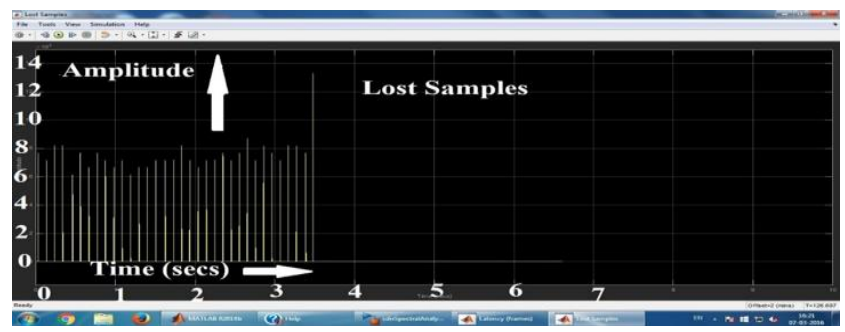

(d)

Figure 3 shows the output results of software defined radio based multiband transceiver. Figure 3(a) \& (b) shows the output power plot in the frequency domain of spectrogram and spectrum waveform.
Figure 3(c) shows latency (frame) output indicate latency during hardware transfer in a number of frames. Figure 3(d) shows lost samples parameters which indicate the number of lost samples during host hardware data transfer. Output data type may be single, double precision floating point or 16 bit signed integers.

\section{CONCLUSION}

Different types of signals are transmitted through the multiband transceiver. Input signals are generated using vector signal generator SMU200A with input frequency range $100 \mathrm{KHz}$ up to 3 GHz. Signals are received using RTL 820T2 SDR dongle which ranges from $25 \mathrm{MHz}$ to $1800 \mathrm{MHz}$ (approximately) with an span of $25 \mathrm{MHz}$. The circuit is verified in actual time approach and is established to be functioning reasonably. Alteration in minor latency and amplitude level which might be due to the broadcast faults .On the basis of lost samples and latency, we conclude that different types of data are effectively and proficiently transmitted using software defined based multiband transceiver.

\section{REFERENCES}

[1] Bastian Bloessl,et al : "Towards an Open Source IEEE 802.11p Stack:A Full SDR-based transceiver in GNU Radio”IEEE Vehicular Networking Conference, 2013.

[2] Bo Zhou et al.: "A Reconfigurable FM-UWB Transceiver for short -range wireless communications",IEEE microwave and wireless components letters, VOL, 23,NO 1531-1309 7 July 2013 .digital object identifier 10.1109/LMWC.2013.2264657.

[3] Chi-Yuan Chen,et al : "Reconfigurable Software Defined Radio and its Applications"Tamkang Journal of Science and Engineering, Vol 13,No 1,pp29-38 (2010).

[4] Hooman darabi "Highly Integrated and Tunable RF Front Ends for Reconfigurable Multi-Band Transceivers" IEEE Custom Integrated Circuits Conference2010.

[5] LMS6002D,Multi-band,Multi-standrad Transceiver with Integrated dual DACs and ADCs, www.limemicro.com

[6] Rehan Muzammil, et al: "A Dynamically Reconfigurable Transceiver for Software Defined Radio",International Journal of Computer Applications(0975-8887)Volume 76No.17,August 2013.

[7] Ryynanen J., Kivekasl K., Jussila J. , Parssinen A., Halonen K.: " Direct conversion receiver for GSM900, DCS1800, PCS1900, and WCDMA"Electronics, Circuits and Systems, 2003. ICECS 2003. Proceedings of the 2003 10th IEEE International Conference on (Volume: 2).

[8] Sofoklis plevridis,el al: “A $65 \mathrm{~nm} 3 \mathrm{G}$ Femtocell Multiband Transceiver”, RMO3A-2 ,2014 IEEE Radio Frequency Integrated Circuits Symposium.

[9] Summary of Characteristics of some leading WLAN/WPAN standards.page no 68: IEEE Communication Magazine,July 2003.

[10] Zheng Song,Nan Qi,Baoyong Chi,Zhihua Wang: “A multimode reconfigurable analog baseband with I/Q calibration for GNSS receivers", 19th Asia and South Pacific Design Automation Conference,ASP-DAC 2014: page no 29-30. 